Yalçın Öztürk*

\title{
An efficient numerical algorithm for solving system of Lane-Emden type equations arising in engineering
}

https://doi.org/10.1515/nleng-2018-0062

Received March 26, 2018; revised May 11, 2018; accepted August 3, 2018.

\begin{abstract}
The purpose of this paper is to propose an efficient numerical method for solving system of LaneEmden type equations using Chebyshev operational matrix method. This method transforms the system of LaneEmden type equation into the system of algebraic equations with unknown Chebyshev coefficients. Some illustrative examples are given to demonstrate the efficiency and validity of the proposed algorithm.
\end{abstract}

Keywords: Lane-Emden system; engineering problem; Chebyshev polynomials; operational matrix method

\section{Introduction}

In this paper, we consider the system of the Lane-Emden type equations as:

$$
\begin{aligned}
& P(t) \frac{d^{2} y_{1}(t)}{d t^{2}}+\frac{\alpha}{t} \frac{d y_{1}(t)}{d t}+y_{2}^{p}(t)=g_{1}(t) \\
& H(t) \frac{d^{2} y_{2}(t)}{d t^{2}}+\frac{\beta}{t} \frac{d y_{2}(t)}{d t}+y_{1}^{q}(t)=g_{2}(t)
\end{aligned}
$$

with initial conditions

$$
\begin{aligned}
& y_{1}(0)=\lambda_{0}, \quad y_{1}^{\prime}(0)=\lambda_{1} \\
& y_{2}(0)=y_{0}, \quad y_{2}^{\prime}(0)=y_{1}
\end{aligned}
$$

where $p, q$ are positive integers. Eq. (1) is the Lane-Emden equation of the first kind, or of index $q$, which is a basic equation in the theory of stellar structure, in the sequence of papers [1-28]. The Lane-Emden equation of the first kind describes the temperature variation of a spherical gas cloud under the mutual attraction of its molecules and subject to the laws of thermodynamics. Notice that the

\footnotetext{
*Corresponding Author: Yalçın Öztürk, Ula Ali Koçman Vocational High School, Muğla Sıtkı Koçman University, Muğla, Turkey, E-mail: yozturk@mu.edu.tr
}

Lane-Emden equation is linear for $q=0,1$ and nonlinear otherwise. In astrophysics, the Lane-Emden equation is Poisson's equation for the gravitational potential of a selfgravitating spherically symmetric and polytropic fluid at hydrostatic equilibrium for [22]. Moreover, one of the important fields of application of this equation is the analysis of the diffusive transport and chemical reaction of species inside a porous catalyst particle [22].

The modelling of several physical phenomea such as pattern formation, population evolution, chemical reactions, and so on [7] gives rise to the systems of Lane-Emden equations, and have attracted much attention in recent years. Several authors have proved existence and uniqueness results for the Lane-Emden systems [8, 9].

Several methods for the solution of Lane-Emden equations and the system of Lane-Emden equations have been presented, which are sinc-collocation method [1], the variational iteration method [2], collocation method [10], the modified Homotopy analysis method [11], the variational iteration method [12], the Adomian decomposition method [13], Legendre operational matrix method [14], second kind Chebyshev operational algorithm [15], ultraspherical wavelets method [16], shifted Legendre operational matrix method [17], spectral second kind Chebyshev wavelets [18] other methods, in the sequence of papers [19-30].

Chebyshev polynomials are the most famous bases of polynomials space. It is well known that there are four kinds of Chebyshev polynomials, and all of them are special cases of the more widest class of Jacobi polynomials. These polynomials have reliable advantage such as easy to compute, rapid convergence and completeness, since Chebyshev polynomials (first kind) is the Fourier cosine series [31, 32]. From this reasons, we use the first kind shifted Chebyshev polynomials in approximation.

Spectral methods play prominent roles in numerical mathematics to solve many more important problems. In this article, we use the operational method which is the kind of spectral method. Operational method have become increasingly popular to solve various problems in applied mathematics. A large number of authors utilize 
this method to solve various equations such as linear and nonlinear hyperbolic telegraph type equations [33], fractional order differential equation [34], higher-order ordinary differential equations [35, 36], Abel equation [37], two point boundary value problems [38], singularly perturbed boundary value problems of fractional multi-order [39], integro-differential equation [40].

The aim of this study is to get solution as truncated Chebyshev series defined by

$$
y_{1}^{N}(t)=\sum_{n=0}^{N}{ }^{\prime} a_{n} T_{n}^{\star}(t), y_{2}^{N}(t)=\sum_{n=0}^{N}{ }^{\prime} b_{n} T_{n}^{\star}(t)
$$

where $T_{n}^{\star}(t)=\cos (n \theta), 2 t-1=\cos \theta, 0 \leq t \leq 1$, denotes the shifted Chebyshev polynomials of the first kind; $\sum^{\prime}$ denotes a sum whose first term is halved; $a_{n}, b_{n}(0 \leq n \leq N)$ are unknown Chebyshev coefficients, and $N$ is chosen any positive integer such that $N \geq m$.

\section{The first kind shifted Chebyshev polynomials}

The Chebyshev polynomials of the first kind $T_{n}(x)$ is a polynomials in $x$ of degree $n$, defined by relation $[31,32]$

$$
T_{n}(x)=\cos n \theta \text {, when } x=\cos \theta
$$

If the range of the variable $x$ is the interval $[-1,1]$, the range the corresponding variables $\theta$ can be taken $[0, \pi]$. We map the independent variable $t$ in $[0,1]$ to the variable $s$ in $[-1,1]$ by transformation

$$
s=2 x-1 \text { or } x=\frac{1}{2}(s+1)
$$

and this lead to the shifted Chebyshev polynomial of the first kind $T_{n}^{\star}(x)$ of degree $n$ in $x$ on [0,1] given by [31]

$$
T_{n}^{\star}(x)=T_{n}(s)=T_{n}(2 x-1) .
$$

These polynomials may be generated by using recurrence relation

$$
T_{n}^{\star}(x)=2(2 x-1) T_{n-1}^{\star}(x)-T_{n-2}^{\star}(x)
$$

with initial conditions

$$
T_{0}^{\star}(x)=1, T_{1}^{\star}(x)=2 x-1,
$$

Any given function, $y(x) \in L^{2}[0,1]$, can be approximated as a sum of shifted Chebyshev polynomials as:

$$
y(x)=\sum_{n=0}^{\infty} a_{n} T_{n}^{\star}(x)
$$

where

$$
c_{n}=\left\langle y(x), T_{n}^{\star}(x)\right\rangle=\int_{0}^{1} y(x) T_{n}^{\star}(x) d x, n=0,1, \ldots, .
$$

\section{Fundamental relations}

Let us consider Eq. (1) and find the matrix forms of the equation. First we can convert the solution $y_{1,2}^{N}(t)$ defined by a truncated shifted Chebyshev series (3) and its derivative $\left(y_{1,2}^{N}(t)\right)^{(k)}$ to matrix forms

$$
\begin{gathered}
y_{1}^{N}(t)=\mathbf{T}^{\star}(t) \mathbf{A},{ }_{1}^{N^{(k)}}(t)=\mathbf{T}^{\star(k)}(t) \mathbf{A}, k=0,1,2 \\
y_{2}^{N}(t)=\mathbf{T}^{\star}(t) B, y_{2}^{N(k)}(t)=\mathbf{T}^{\star(k)}(t) \mathbf{B}, k=0,1,2
\end{gathered}
$$

where

$$
\begin{aligned}
& \mathbf{T}^{\star}(t)=\left[T_{0}^{\star}(t) T_{1}^{\star}(t) \ldots T_{N}^{\star}(t)\right] \mathbf{T}^{\star(k)}(t) \\
& =\left[T_{0}^{\star(k)}(t) T_{1}^{\star(k)}(t) \ldots T_{N}^{\star(k)}(t)\right] \\
& \mathbf{A}=\left[\begin{array}{llll}
\frac{1}{2} a_{0} & a_{1} \ldots & a_{N}
\end{array}\right]^{T} \\
& \mathbf{B}=\left[\frac{1}{2} b_{0} b_{1} \ldots b_{N}\right]^{T}
\end{aligned}
$$

It is well known that the relation between the powers $x^{n}$ and the shifted Chebyshev polynomials $T_{n}^{\star}(x)$ is

$$
x^{n}=2^{-2 n+1} \sum_{k=0}^{n},\left(\begin{array}{l}
2 n \\
k
\end{array}\right) T_{n-k}^{\star}(x), 0 \leq x \leq 1
$$

where $\sum^{\prime}$ denotes a sum whose first term is halved.

By using the expression (6) and taking $n=0,1, \ldots, N$ we find the corresponding matrix relation as follows:

$$
(\mathbf{X}(t))^{T}=\mathbf{D}\left(\mathbf{T}^{\star}(t)\right)^{T} \quad \text { and } \quad \mathbf{X}(t)=\mathbf{T}^{\star}(t) \mathbf{D}^{T}
$$

where 


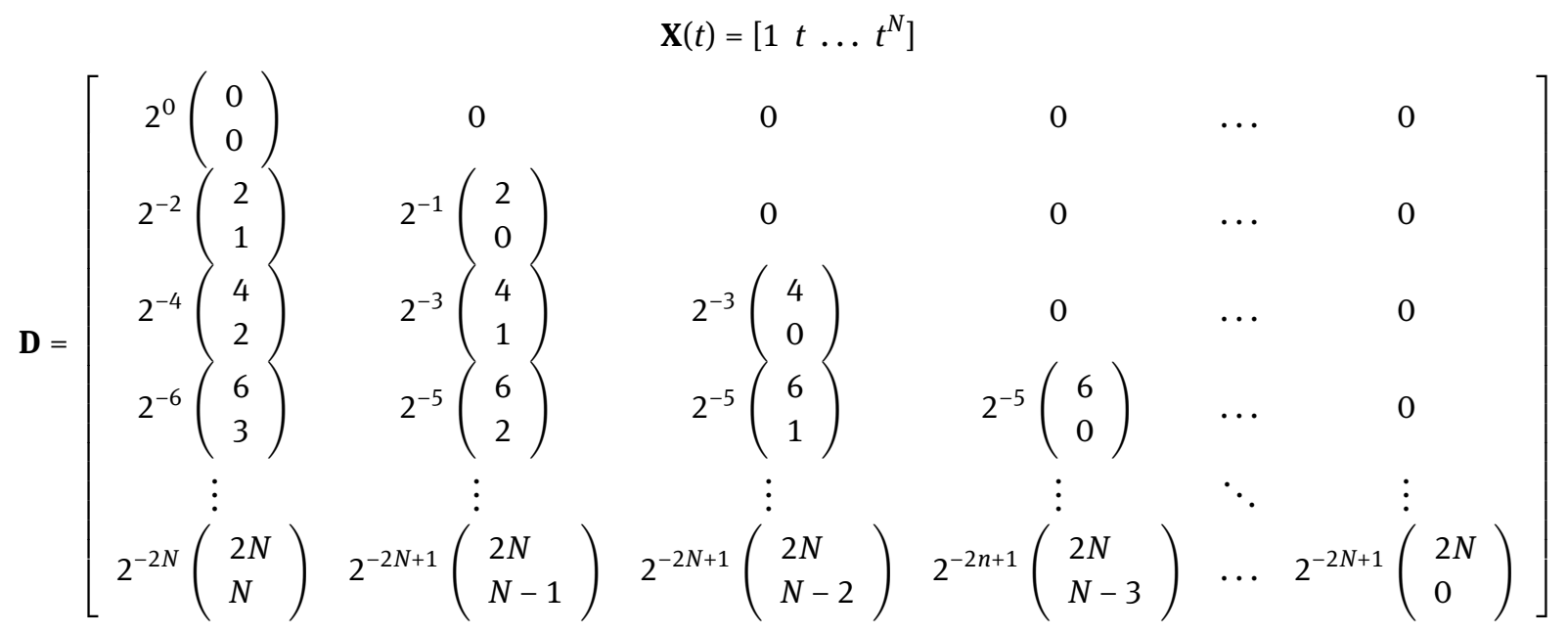

Then, by taking into account (7) we obtain

$$
\mathbf{T}^{\star}(t)=\mathbf{X}(t)\left(\mathbf{D}^{-1}\right)^{T}
$$

and

$$
\left(\mathbf{T}^{\star}(t)\right)^{(k)}=\mathbf{X}^{(k)}(t)\left(\mathbf{D}^{-1}\right)^{T}, k=0,1,2,
$$

To obtain the matrix $\mathbf{X}^{(k)}(t)$ in terms of the matrix $\mathbf{X}(t)$, we can use the following relation:

$$
\begin{gathered}
\mathbf{X}^{(1)}(t)=\mathbf{X}(t) \mathbf{C} \\
\mathbf{X}^{(2)}(t)=\mathbf{X}^{(1)}(t) \mathbf{C}=\mathbf{X}(t)(\mathbf{C})^{2}
\end{gathered}
$$

where

$$
\mathbf{C}=\left[\begin{array}{ccccc}
0 & 0 & 0 & \ldots & 0 \\
1 & 0 & 0 & \ldots & 0 \\
0 & 2 & 0 & \ldots & 0 \\
\ldots & \ldots & \ldots & \ldots & \ldots \\
0 & 0 & 0 & N & 0
\end{array}\right]
$$

Consequently, by substituting the matrix forms (8) and (9) into (4)-(5) we have the matrix relation

$$
\begin{aligned}
& y_{1}^{N}(t)^{(k)}=\mathbf{X}(t) \mathbf{C}^{k}\left(\mathbf{D}^{T}\right)^{-1} \mathbf{A}, k=0,1,2 \\
& y_{2}^{N}(t)^{(k)}=\mathbf{X}(t) \mathbf{C}^{k}\left(\mathbf{D}^{T}\right)^{-1} \mathbf{B}, k=0,1,2
\end{aligned}
$$

\section{Method of Solution}

Firstly, we deal with singularity in Eq. (1). If they set in Eq. (1) by [16]

$$
g_{1}(t)=t f_{1}(t) g_{2}(t)=t f_{2}(t),
$$

Then Eq. (1) is turned into

$$
\begin{aligned}
& t P(t) \frac{d^{2} y_{1}(t)}{d t^{2}}+\alpha \frac{d y_{1}(t)}{d t}+t y_{2}^{p}(t)=f_{1}(t) \\
& t H(t) \frac{d^{2} y_{2}(t)}{d t^{2}}+\beta \frac{d y_{2}(t)}{d t}+t y_{1}^{q}(t)=f_{2}(t)
\end{aligned}
$$




$$
\begin{aligned}
& y_{1}(0)=\lambda_{0}, \quad y_{1}^{\prime}(0)=\lambda_{1} \\
& y_{2}(0)=y_{0}, \quad y_{2}^{\prime}(0)=y_{1}
\end{aligned}
$$

Now, we present the numerical solution method for Eq. (12) with initial conditions Eq. (13). We can give the expansion of $f_{i}(x)$ by the shifted Chebyshev polynomials as:

$$
f_{i}(t) \approx \mathbf{F}_{\mathbf{i}}^{\mathbf{T}} \mathbf{X}(t)\left(\mathbf{D}^{T}\right)^{-1}, i=1,2
$$

Using matrix representation of approximate solution and its derivatives, Eq. (12) can be written as:

$$
\begin{aligned}
& t P(t) \mathbf{X}(t) \mathbf{C}^{2}\left(\mathbf{D}^{T}\right)^{-1} \mathbf{A}+\alpha \mathbf{X}(t) \mathbf{C}\left(\mathbf{D}^{T}\right)^{-1} \mathbf{A}+t\left(\mathbf{X}(t)\left(\mathbf{D}^{T}\right)^{-1} \mathbf{B}\right)^{\mathbf{p}} \\
& =f_{1}(t)
\end{aligned}
$$

$t H(t) \mathbf{X}(t) \mathbf{C}^{2}\left(\mathbf{D}^{T}\right)^{-1} \mathbf{B}+\beta \mathbf{X}(t) \mathbf{C}\left(\mathbf{D}^{T}\right)^{-1} \mathbf{B}+t\left(\mathbf{X}(t)\left(\mathbf{D}^{T}\right)^{-1} \mathbf{A}\right)^{\mathbf{q}}$ $=f_{2}(t)$

The residual $R_{i}(t)$ for Eq. (15) can be written as

$$
\begin{aligned}
& R_{1}(t) \approx t P(t) \mathbf{X}(t) \mathbf{C}^{2}\left(\mathbf{D}^{T}\right)^{-1} \mathbf{A}+\alpha \mathbf{X}(t) \mathbf{C}\left(\mathbf{D}^{T}\right)^{-1} \mathbf{A} \\
& +t\left(\mathbf{X}(t)\left(\mathbf{D}^{T}\right)^{-1} \mathbf{B}\right)^{\mathbf{p}}-f_{1}(t) \\
& R_{2}(t) \approx t H(t) \mathbf{X}(t) \mathbf{C}^{2}\left(\mathbf{D}^{T}\right)^{-1} \mathbf{B}+\beta \mathbf{X}(t) \mathbf{C}\left(\mathbf{D}^{T}\right)^{-1} \mathbf{B} \\
& +t\left(\mathbf{X}(t)\left(\mathbf{D}^{T}\right)^{-1} \mathbf{A}\right)^{\mathbf{q}}-f_{2}(t)
\end{aligned}
$$

and if it is applied typical tau method which is used in the sense of a particular form of the Petrov-Galerkin method [33-40], Eqs. (16) - (17) can be converted in $2 \times$ $(N-1)$ nonlinear equations by calculating

$$
\left\langle R_{i}(t), T_{n}^{\star}(t)\right\rangle=\int_{0}^{1} R_{i}(t) T_{n}^{\star}(t) d t=0, n=0,1, \ldots, N-1
$$

The initial conditions (13) lead to the four equations

$$
\begin{aligned}
& y_{1}^{N}(0)=\mathbf{X}(0)\left(\mathbf{D}^{T}\right)^{-1} \mathbf{A}=\lambda_{0}, \\
& \left(y_{1}^{N}(x)\right)^{\prime}(0)=\mathbf{X}(0) \mathbf{C}\left(\mathbf{D}^{T}\right)^{-1} \mathbf{A}=\lambda_{1} \\
& y_{2}^{N}(0)=\mathbf{X}(0)\left(\mathbf{D}^{T}\right)^{-1} \mathbf{B}=y_{0}, \\
& \left(y_{2}^{N}\right)^{\prime}(0)=\mathbf{X}(0) \mathbf{C}\left(\mathbf{D}^{T}\right)^{-1} \mathbf{B}=y_{1}
\end{aligned}
$$

Therefore, we get the $2 \times(N+1)$ sets of equations with $2 \times(N+1)$ unknowns by Eq. (18) and Eqs. (19) - (20). We write the program in the Maple 13 and solve the $2 \times(N+1)$ sets of equations with $2 \times(N+1)$ unknowns, and so approximate solution $y_{i}^{N}(x), i=1,2$ can be calculated.

\subsection{Error estimation and convergence analysis}

Assume that $H=L^{2}[0,1]$, where $P_{N}=$ $\left\{T_{0}^{\star}(t), T_{1}^{\star}(t), \ldots, T_{N}^{\star}(t)\right\} \subset H$ be the set of polynomials of $n-t h$ degree and $W=\operatorname{Span}\left(P_{N}\right)$. Clearly, $W$ is a finite dimensional vector space. Let $f \in H$, then $f$ has the unique best approximation out of $W$ such that $g_{0} \in W$, that is [41]

$$
\left\|f-g_{0}\right\|_{2} \leq\|f-g\|_{2}, \forall g \in W
$$

where $\|f\|_{2}^{2}=<f, f>$. There exist unique coefficients $\mathbf{A}=$ $\left[\begin{array}{llll}a_{0} & a_{1} \ldots & a_{N}\end{array}\right]$ for $g_{0}$ such that

$$
f \approx g_{0}=\sum_{k=0}^{N} a_{k} T_{k}^{\star}(t)=\mathbf{T}(t) \mathbf{A}
$$

where $\mathbf{T}(x)=\left[T_{0}^{\star}(t), T_{1}^{\star}(t), \ldots, T_{N}^{\star}(t)\right]$ and coefficient matrix $\mathbf{A}$ can be given by the following equation

$$
\mathbf{A}<\mathbf{T}(t), \mathbf{T}(t)>=\langle f, \mathbf{T}(t)>
$$

where

$$
\begin{aligned}
& <f, \mathbf{T}(t)>=\int_{0}^{1} f(t) \mathbf{T}(t)^{T} d x= \\
& {\left[<f, T_{0}^{\star}(t)><f, T_{1}^{\star}(t)>\ldots<f, T_{N}^{\star}(t)>\right]}
\end{aligned}
$$

and $<\mathbf{T}(t), \mathbf{T}(t)>$ is an $(N+1) \times(N+1)$ matrix and

$$
\phi=<\mathbf{T}(t), \mathbf{T}(t)>=\int_{0}^{1} \mathbf{T}(t) \mathbf{T}(t)^{T} d t
$$

and so

$$
\mathbf{A}=\left(\int_{0}^{1} f(t) \mathbf{T}(t)^{T} d t\right) \phi^{-1}
$$

Theorem 1. [41] Let assume that $H$ is an Hilbert space, $W$ is a closed subspace of $H$ such that $\operatorname{dim} W$ is finite and $\left\{y_{1}, y_{2}, y_{3}, \ldots, y_{N}\right\}$ is an y basis for $W$. Let $f$ be an arbitrary element in $H$ and $g_{0}$ be the unique best approximation to $f$ out of $W$. Then we have

$$
\left\|f-g_{0}\right\|_{2}^{2}=\frac{D\left(f, y_{1}, y_{2}, \ldots, y_{N}\right)}{D\left(y_{1}, y_{2}, \ldots, y_{N}\right)}
$$

where

$$
\begin{aligned}
& D\left(f, y_{1}, y_{2}, \ldots, y_{N}\right)= \\
& \left|\begin{array}{cccc}
<f, f> & <f, y_{1}> & \cdots & <f, y_{N}> \\
<y_{1}, f> & <y_{1}, y_{1}> & \cdots & <y_{1}, y_{N}> \\
\vdots & \vdots & \ddots & \vdots \\
<y_{N}, f> & <y_{N}, y_{1}> & \cdots & <y_{N}, y_{N}>
\end{array}\right|
\end{aligned}
$$



$\int_{a}^{b} f(t) g(t)$ and the subspace $W=\operatorname{Span}\left(P_{N}\right)$, so the presented absolute error in Theorem 1 can be written

$$
\left\|f-g_{0}\right\|=\frac{\operatorname{det}\left(\int_{0}^{1} \Psi(t) \Psi(t)^{T} d t\right)}{\operatorname{det}\left(\int_{0}^{1} \Phi(t) \Phi(t)^{T} d t\right)}
$$

for which $\Phi(t)^{T}=\left[T_{0}^{\star}(t) T_{1}^{\star}(t) \ldots T_{N}^{\star}(t)\right]$ and $\Psi(x)^{T}=$ $\left[f T_{0}^{\star}(t) T_{1}^{\star}(t) \ldots T_{N}^{\star}(t)\right]$.

Theorem 2. Assume that the function $g:[0,1] \rightarrow R$ is $(N+1)$ times continuously differentiable, $g \in C^{N+1}[0,1]$ and $W=\operatorname{Span}\left\{T_{0}^{\star}(t), T_{1}^{\star}(t), \ldots, T_{N}^{\star}(t)\right\}$. If $\mathbf{A} \Phi$ is the best approximation to $g$ out of $W$, then a bound for absolute error is presented by

$$
\|g-\mathbf{A} \Phi\| \leq \frac{M^{2}}{2^{2 N}((N+1) !)^{2}}
$$

where $M=\max _{x \in[0,1]}\left(g_{i}(t)^{(N+1)}\right)$.

Proof. We consider the interpolation polynomial $g^{\star}(t)$ is the interpolating polynomial to $g$ at $t_{i}$, where $t_{i}, i=$ $0,1, \ldots, N$ are the Chebyshev-Gauss grid points, then we have

$$
g(t)-g^{\star}(t)=\frac{g^{(N+1)}(\lambda)}{(N+1) !} \prod_{i=0}^{N}\left(t-t_{i}\right), \lambda \in[0,1]
$$

Since, $\left\|T_{N}^{\star}(t)\right\|_{\infty}=1$, we conclude that if we choose the grid nodes $\left(t_{i}\right)_{0 \leq i \leq N}$ to be zero the $(\mathrm{N}+1)$ zeroes of the Chebyshev polynomials $T_{N}^{\star}(t)$, we have $[25,26]$

$$
\left\|\prod_{i=0}^{N}\left(t-t_{i}\right)\right\|=\frac{1}{2^{N}}
$$

this is the smallest possible value. From (25), we obtain

$$
\left\|g(t)-g^{\star}(t)\right\|_{\infty} \leq \frac{1}{2^{N}(N+1) !}\left\|g(t)^{(N+1)}\right\|_{\infty} .
$$

Since $\mathbf{A} \Phi$ is the best approximation to $g$ out of $W$, considering $g^{\star} \in W$ and using (28), we have

$$
\begin{aligned}
& \|g-\mathbf{A} \Phi\| \leq\left\|g-g^{\star}\right\|=\int_{0}^{1}\left|g(t)-g^{\star}(t)\right|^{2} d t \\
& \leq \int_{0}^{1}\left(\frac{M}{2^{N}(N+1) !}\right)^{2} d x \leq \frac{M^{2}}{2^{2 N}((N+1) !)^{2}}
\end{aligned}
$$

Another comparison can be given for quality of the approximation by well known algorithm [32]. Since the approximate solution $y_{1,2}^{N}(t)$ is the approximate solution of
Eq. (1), Eq. (1) must be approximately satisfied by the function $y_{N}(x)$, that is;

$$
\begin{aligned}
& \left|P(t) \frac{d^{2} y_{1}^{N}(t)}{d t^{2}}+\frac{\alpha}{t} \frac{d y_{1}^{N}(t)}{d t}+\left(y_{2}^{N}(t)\right)^{p}-g_{1}(t)\right| \approx 0 \\
& \left|H(t) \frac{d^{2} y_{2}^{N}(t)}{d t^{2}}+\frac{\beta}{t} \frac{d y_{2}^{N}(t)}{d t}+\left(y_{1}^{N}(t)\right)^{q}-g_{2}(t)\right| \approx 0
\end{aligned}
$$

This comparison should be advised strongly by [32]. Then the error can be estimated by the error function [32]

$$
\begin{aligned}
& E_{1}^{N}=\left|P(t) \frac{d^{2} y_{1}^{N}(t)}{d t^{2}}+\frac{\alpha}{t} \frac{d y_{1}^{N}(t)}{d t}+\left(y_{2}^{N}(t)\right)^{p}-g_{1}(t)\right| \\
& E_{2}^{N}=\left|H(t) \frac{d^{2} y_{2}^{N}(t)}{d t^{2}}+\frac{\beta}{t} \frac{d y_{2}^{N}(t)}{d t}+\left(y_{1}^{N}(t)\right)^{q}-g_{2}(t)\right|
\end{aligned}
$$

\section{Examples}

In this section, several numerical examples are given to illustrate the accuracy and effectiveness properties of the method and all of them were performed on the computer using a program written in Maple 13. Let us define

$$
E_{i}^{N, L}=\left(\int_{0}^{1}\left(y(t)-y_{N}(t)\right)^{2} d t\right)^{1 / 2}
$$

where $y_{i}(x)$ and $y_{i}^{N}(x), i=1,2$ denote the approximate solution obtained by the present method and the exact solution, respectively. In Tables, $N_{e}=\left|y_{i}(t)-y_{i}^{N}(t)\right|$ are absolute error for selected points.

Example 1. Let us consider the following linear systems of Lane-Emden equations [26]

$$
\begin{aligned}
& y_{1}^{\prime \prime}(t)+\frac{3}{t} y_{1}^{\prime}(t)-4\left(y_{1}(t)+y_{2}(t)\right)=0 \\
& y_{2}^{\prime \prime}(t)+\frac{2}{t} y_{2}^{\prime}(t)+3\left(y_{1}(t)+y_{2}(t)\right)=0
\end{aligned}
$$

subject to initial conditions

$$
y_{1}(0)=1, y_{2}(0)=1, y_{1}^{\prime}(0)=0, y_{2}^{\prime}(0)=0 \text {. }
$$

For $N=2$, We have the residual $R_{i}^{N}(t)$ for this problem

$$
\begin{aligned}
& \mathbf{R}_{1} \approx t \mathbf{X}(t) \mathbf{C}^{2}\left(\mathbf{D}^{T}\right)^{-1} \mathbf{A}+3 \mathbf{X}(t) \mathbf{C}\left(\mathbf{D}^{T}\right)^{-1} \mathbf{A}-4 t \mathbf{X}(t)\left(\mathbf{D}^{T}\right)^{-1} \mathbf{A} \\
& -4 t \mathbf{X}(t)\left(\mathbf{D}^{T}\right)^{-1} \mathbf{B}-\mathbf{F}_{\mathbf{1}}^{\mathbf{T}} \mathbf{X}(t)\left(\mathbf{D}^{T}\right)^{-1}
\end{aligned}
$$

$$
\begin{aligned}
& \mathbf{R}_{2} \approx t \mathbf{X}(t) \mathbf{C}^{2}\left(\mathbf{D}^{T}\right)^{-1} \mathbf{B}+2 \mathbf{X}(t) \mathbf{C}\left(\mathbf{D}^{T}\right)^{-1} \mathbf{B}+3 t \mathbf{X}(t)\left(\mathbf{D}^{T}\right)^{-1} \mathbf{A} \\
& +3 t \mathbf{X}(t)\left(\mathbf{D}^{T}\right)^{-1} \mathbf{B}-\mathbf{F}_{\mathbf{2}}^{\mathbf{T}} \mathbf{X}(t)\left(\mathbf{D}^{T}\right)^{-1}
\end{aligned}
$$


where

$$
\begin{gathered}
\mathbf{D}=\left[\begin{array}{ccc}
1 & 0 & 0 \\
1 / 2 & 1 / 2 & 0 \\
3 / 8 & 1 / 2 & 1 / 8
\end{array}\right] \mathbf{C}=\left[\begin{array}{lll}
0 & 1 & 0 \\
0 & 0 & 2 \\
0 & 0 & 0
\end{array}\right] \\
\mathbf{X}(x)=\left[\begin{array}{c}
1 \\
x \\
x^{2}
\end{array}\right] \mathbf{F}_{\mathbf{1}}=\left[\begin{array}{l}
0 \\
0 \\
0
\end{array}\right] \\
\mathbf{F}_{2}=\left[\begin{array}{l}
0 \\
0 \\
0
\end{array}\right] \mathbf{A}=\left[\begin{array}{l}
a_{0} \\
a_{1} \\
a_{2}
\end{array}\right] \mathbf{B}=\left[\begin{array}{l}
b_{0} \\
b_{1} \\
b_{2}
\end{array}\right]
\end{gathered}
$$

Then, using Eqs.(34)-(35) we obtain the linear algebraic system

$$
\begin{aligned}
& -2 a_{0}+\frac{16}{3} a_{1}+\frac{26}{3} a_{2}-2 b_{0}-\frac{2}{3} b_{1}+\frac{2}{3} b_{2}=0 \\
& \frac{3}{2} a_{0}+\frac{1}{2} a_{1}-\frac{1}{2} a_{2}+\frac{3}{2} b_{0}+\frac{9}{2} b_{1}+\frac{15}{2} b_{2}=0
\end{aligned}
$$

with conditions

$$
\begin{gathered}
y_{1}^{2}(0)=\mathbf{X}(0)\left(\mathbf{D}^{T}\right)^{-1} \mathbf{A}=a_{0}-a_{1}+a_{2}=1 \\
y_{2}^{2}(0)=\mathbf{X}(0)\left(\mathbf{D}^{T}\right)^{-1} \mathbf{B}=2 a_{1}-8 a_{2}=0 \\
\left(y_{1}^{2}(x)\right)^{\prime}(0)=\mathbf{X}(0) \mathbf{C}\left(\mathbf{D}^{T}\right)^{-1} \mathbf{A}=b_{0}-b_{1}+b_{2}=1 \\
\left(y_{2}^{2}(x)\right)^{\prime}(0)=\mathbf{X}(0) \mathbf{C}\left(\mathbf{D}^{T}\right)^{-1} \mathbf{A}_{2}=2 b_{1}-8 b_{2}=0
\end{gathered}
$$

Solving Eqs. (36) - (41), we obtain

$$
\begin{gathered}
a_{0}=1.375, a_{1}=0.5, a_{2}=0.125 \\
b_{0}=0.625, b_{1}=-0.5, b_{2}=-0.125
\end{gathered}
$$

Subsituting these coefficients in Eqs. (4) - (5), then we get

$$
\begin{aligned}
& y_{1}^{2}(x)=1+t^{2} \\
& y_{2}^{2}(x)=1-t^{2}
\end{aligned}
$$

These solutions are exact solution of this problem.

Example 2. Let us consider the linear, nonhomogeneous systems of Lane-Emden equations [26]

$$
\begin{aligned}
& y_{1}^{\prime \prime}(t)+\frac{2}{t} y_{1}^{\prime}(t)-\left(4 t^{2}+6\right) y_{1}(t)+y_{2}(t)=t^{4}-t^{3} \\
& y_{2}^{\prime \prime}(t)+\frac{8}{t} y_{2}^{\prime}(t)+t y_{2}(t)+y_{1}(t)=e^{t^{2}}+t^{5}-t^{4}+44 t^{2}-30 t
\end{aligned}
$$

subject to conditions

$$
y_{1}(0)=1, y_{1}^{\prime}(0)=0, y_{2}(0)=0, y_{2}^{\prime}(0)=0
$$

The comparison among present method and exact solution are shown in Table 1 and Table 2. In Table 3, the computational results of the $L^{2}$-norm error between the approximate solutions and exact solution for different $N$ and truncated errors are summarized. Figs. 1 - 2 represent the error between exact and approximate solutions for $N=6,8$. These figures show that errors are descreasing when $N$ increasing.

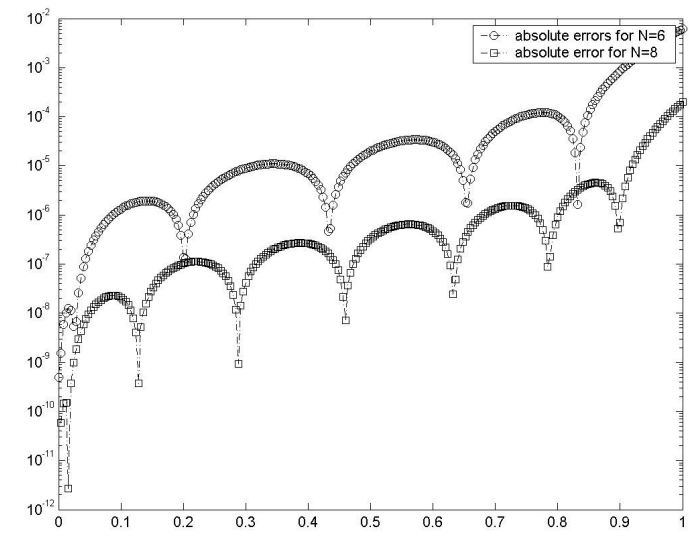

Fig. 1: Comparison of absolute errors function for $y_{1}(t)$

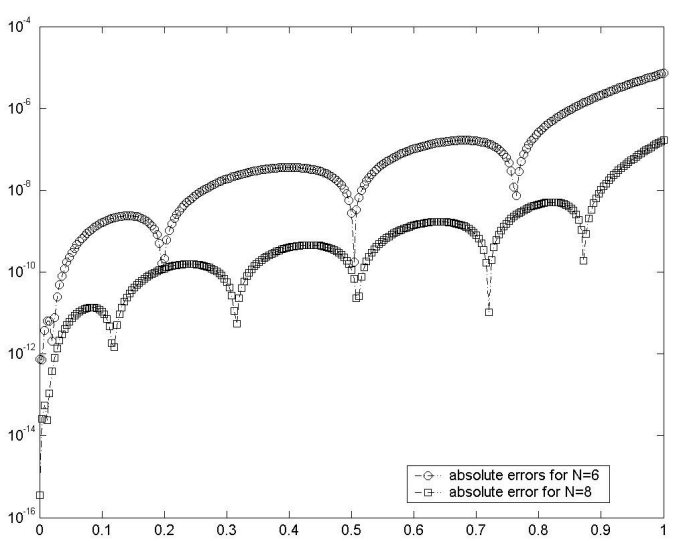

Fig. 2: Comparison of absolute errors function for $y_{2}(t)$

Example 3. Let us consider the nonlinear equation [26]

$$
\begin{aligned}
& y_{1}^{\prime \prime}(t)+\frac{5}{t} y_{1}^{\prime}(t)+8\left(e^{y_{1}(x)}+2 e^{-y_{2} / 2}\right)=0 \\
& y_{2}^{\prime \prime}(t)+\frac{3}{t} y_{2}^{\prime}(t)-8\left(e^{-y_{2}}+e^{y_{1} / 2}\right)=0
\end{aligned}
$$

with initial conditions

$$
y_{1}(0)=1, y_{1}^{\prime}(0)=0, y_{2}(0)=0, y_{2}^{\prime}(0)=0
$$


Table 1: Numerical result for approximate solution of $y_{1}(t)$ in Example 2.

\begin{tabular}{cccccccc}
\hline$t$ & Exact Solution & $\mathrm{N}=5$ & $\mathrm{~N}_{e}=5$ & \multicolumn{2}{c}{$\mathrm{N}=6 \mathrm{~N}_{e}=6$} & $\mathrm{~N}=8$ & $\mathrm{~N}_{e}=8$ \\
\hline 0.0 & 1.000000 & 0.999999 & $0.800 \mathrm{E}-8$ & 0.999999 & $0.500 \mathrm{E}-9$ & 1.000000 & $0.000 \mathrm{E}-0$ \\
0.2 & 1.040810 & 1.040834 & $0.238 \mathrm{E}-4$ & 1.040810 & $0.135 \mathrm{E}-6$ & 1.040810 & $0.102 \mathrm{E}-6$ \\
0.4 & 1.173510 & 1.173384 & $0.126 \mathrm{E}-3$ & 1.173517 & $0.690 \mathrm{E}-5$ & 1.173510 & $0.261 \mathrm{E}-6$ \\
0.6 & 1.433329 & 1.433539 & $0.209 \mathrm{E}-3$ & 1.433298 & $0.305 \mathrm{E}-4$ & 1.433329 & $0.471 \mathrm{E}-6$ \\
0.8 & 1.896480 & 1.895792 & $0.688 \mathrm{E}-2$ & 1.896583 & $0.102 \mathrm{E}-3$ & 1.896481 & $0.909 \mathrm{E}-6$ \\
1.0 & 2.718281 & 2.686791 & $0.314 \mathrm{E}-1$ & 2.712165 & $0.611 \mathrm{E}-3$ & 2.718083 & $0.197 \mathrm{E}-3$ \\
\hline
\end{tabular}

Table 2: Numerical result for approximate solution of $y_{2}(t)$ in Example 2.

\begin{tabular}{cccccccc}
\hline$t$ & Exact Solution & $\mathrm{N}=5$ & $\mathrm{~N}_{e}=5$ & \multicolumn{2}{c|}{$\mathrm{N}=6 \mathrm{~N}_{e}=6$} & $\mathrm{~N}=8$ & $\mathrm{~N}_{e}=8$ \\
\hline 0.0 & 0.0000 & $0.000 \mathrm{E}-0$ & $0.000 \mathrm{E}-0$ & $0.000 \mathrm{E}-0$ & $0.000 \mathrm{E}-0$ & 0.00000 & $0.000 \mathrm{E}-0$ \\
0.2 & -0.0064 & -0.006400 & $0.436 \mathrm{E}-7$ & -0.006400 & $0.189 \mathrm{E}-9$ & -0.00640 & $0.122 \mathrm{E}-9$ \\
0.4 & -0.0384 & -0.038399 & $0.343 \mathrm{E}-6$ & -0.038400 & $0.358 \mathrm{E}-7$ & -0.03840 & $0.399 \mathrm{E}-9$ \\
0.6 & -0.0864 & -0.086399 & $0.770 \mathrm{E}-5$ & -0.086399 & $0.102 \mathrm{E}-6$ & -0.08640 & $0.138 \mathrm{E}-8$ \\
0.8 & -0.1024 & -0.102400 & $0.620 \mathrm{E}-5$ & -0.102400 & $0.259 \mathrm{E}-6$ & -0.10240 & $0.455 \mathrm{E}-8$ \\
1.0 & 0.0000 & $-0.419 \mathrm{E}-4$ & $0.419 \mathrm{E}-4$ & $-0.722 \mathrm{E}-5$ & $0.722 \mathrm{E}-5$ & $0.168 \mathrm{E}-6$ & $0.168 \mathrm{E}-6$ \\
\hline
\end{tabular}

Table 3: Numerical result for Example 2.

\begin{tabular}{ccccc}
\hline Present method & \multicolumn{2}{c}{$E_{N}^{L}$} & $y_{N}^{T}$ \\
\hline & $y_{1}(t)$ & $y_{2}(t)$ & $y_{1}(t)$ & $y_{2}(t)$ \\
$N=5$ & $0.615773 \times 10^{-2}$ & $101474 \times 10^{-4}$ & $10^{-2}$ & $10^{-6}$ \\
$N=6$ & $0.103500 \times 10^{-2}$ & $0.148370 \times 10^{-5}$ & $10^{-3}$ & $10^{-8}$ \\
$N=8$ & $0.263404 \times 10^{-4}$ & $0.256832 \times 10^{-7}$ & $10^{-5}$ & $10^{-10}$ \\
\hline
\end{tabular}

Above algorithm is applied to this problem, we have $y_{1}(x)=x^{2}-2 \ln 1, y_{2}(x)=x^{2}+2 \ln 1$ which is the exact solution of this problem.

Example 4. Let us consider the following nonlinear problem [26]

$$
\begin{aligned}
& y_{1}^{\prime \prime}(t)+\frac{1}{t} y_{1}^{\prime}(t)-y_{2}^{3}(t)\left(y_{1}^{2}(t)+1\right)=0 \\
& y_{2}^{\prime \prime}(t)+\frac{3}{t} y_{2}^{\prime}(t)+y_{2}^{5}(t)\left(y_{1}^{2}(t)+3\right)=0
\end{aligned}
$$

subject to conditions

$$
y_{1}(0)=1, y_{1}^{\prime}(0)=1, y_{2}(0)=0, y_{2}^{\prime}(0)=0
$$

with exact solutions

$$
y_{1}=\sqrt{1+t^{2}}, \quad y_{2}=\frac{1}{\sqrt{1+t^{2}}},
$$

Applying our method for $N=4,5,6$, obtained numerical results are displayed in Table 4 and Table 5. Also, absolute errors are displayed in Figures 3 and 4. The tables and the figures show that proposed method is in good agreement with the analytical solution.

\section{Conclusion}

An operational matrix method for the solution of systems of Lane-Emden equations has been proposed and investi-

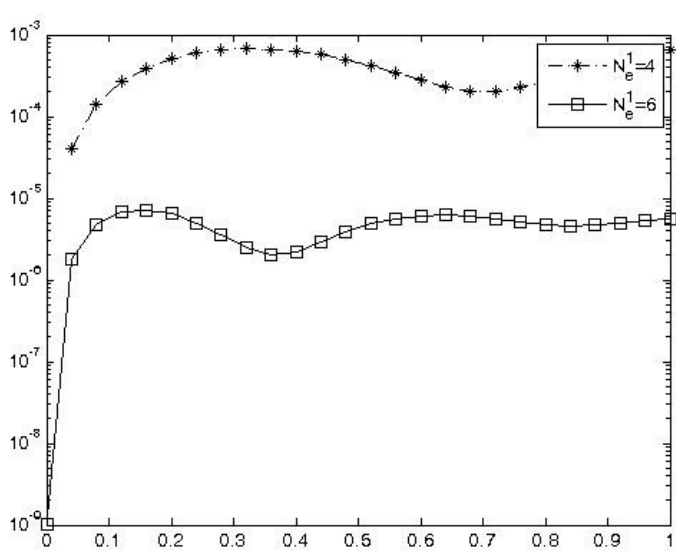

Fig. 3: Comparison of absolute errors function for $y_{1}(t)$ in Ex. 4

gated. Moreover, the convergence and error analysis have been determined. The presented numerical examples have exhibited the high accuracy, applicability, and efficiency of the proposed algorithms. The proposed method has been given to find the analytical solutions if the problem has exact solutions that are polynomial functions. This method has some considerable advantage that only small size operational matrix is required to provide the solution 
Table 4: Numerical result for approximate solution of $y_{1}(t)$ in Example 5.

\begin{tabular}{cccccccc}
\hline$t$ & Exact & \multirow{2}{*}{$\mathrm{N}=4$} & $\mathrm{~N}_{e}=4$ & \multicolumn{2}{c}{$\mathrm{N}=5 \mathrm{~N}_{e}=5$} & $\mathrm{~N}=6$ & $\mathrm{~N}_{e}=6$ \\
\hline 0.0 & 1.00000 & 1.000000 & $0.000 \mathrm{E}-0$ & 1.00000 & $0.000 \mathrm{E}-0$ & 1.00000 & $0.000 \mathrm{E}-0$ \\
0.2 & 1.019803 & 1.020313 & $0.509 \mathrm{E}-3$ & 1.019803 & $0.565 \mathrm{E}-4$ & 1.019803 & $0.642 \mathrm{E}-5$ \\
0.4 & 1.077032 & 1.077661 & $0.628 \mathrm{E}-3$ & 1.077032 & $0.216 \mathrm{E}-4$ & 1.077032 & $0.220 \mathrm{E}-5$ \\
0.6 & 1.166190 & 1.166468 & $0.277 \mathrm{E}-3$ & 1.166190 & $0.557 \mathrm{E}-5$ & 1.166190 & $0.611 \mathrm{E}-5$ \\
0.8 & 1.280624 & 1.280897 & $0.272 \mathrm{E}-3$ & 1.280624 & $0.738 \mathrm{E}-4$ & 1.280624 & $0.471 \mathrm{E}-5$ \\
1.0 & 1.414213 & 1.414858 & $0.644 \mathrm{E}-3$ & 1.414213 & $0.746 \mathrm{E}-4$ & 1.414213 & $0.556 \mathrm{E}-5$ \\
\hline
\end{tabular}

Table 5: Numerical result for approximate solution of $y_{2}(t)$ in Example 5.

\begin{tabular}{cccccccc}
\hline$t$ & Exact Solution & $\mathrm{N}=4$ & $\mathrm{~N}_{e}=4$ & \multicolumn{2}{c}{$\mathrm{N}=5 \mathrm{~N}_{e}=5$} & $\mathrm{~N}=6$ & $\mathrm{~N}_{e}=6$ \\
\hline 0.0 & 1.000000 & 1.000000 & $0.000 \mathrm{E}-0$ & 1.000000 & $0.000 \mathrm{E}-0$ & 1.000000 & $0.000 \mathrm{E}-0$ \\
0.2 & 0.980580 & 0.979703 & $0.887 \mathrm{E}-3$ & 0.980580 & $0.165 \mathrm{E}-4$ & 0.980580 & $0.467 \mathrm{E}-5$ \\
0.4 & 0.928476 & 0.928249 & $0.227 \mathrm{E}-3$ & 0.928476 & $0.151 \mathrm{E}-4$ & 0.928476 & $0.405 \mathrm{E}-5$ \\
0.6 & 0.857492 & 0.858494 & $0.100 \mathrm{E}-3$ & 0.857492 & $0.166 \mathrm{E}-4$ & 0.857492 & $0.185 \mathrm{E}-5$ \\
0.8 & 0.780868 & 0.781561 & $0.692 \mathrm{E}-3$ & 0.780868 & $0.167 \mathrm{E}-4$ & 0.780868 & $0.327 \mathrm{E}-5$ \\
1.0 & 0.707106 & 0.706844 & $0.262 \mathrm{E}-3$ & 0.707106 & $0.608 \mathrm{E}-4$ & 0.707106 & $0.134 \mathrm{E}-6$ \\
\hline
\end{tabular}

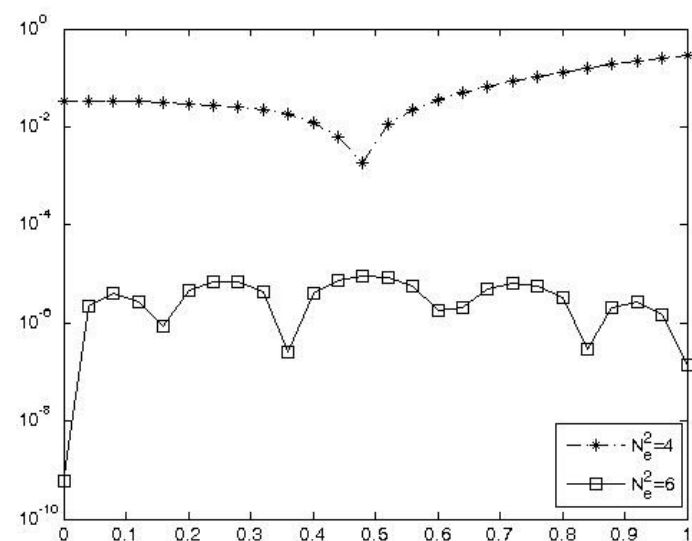

Fig. 4: Comparison of absolute errors for $y_{2}(t)$ in Ex. 4

of high accuracy because most of matrix involves more numbers of zeroes and thus, reduces the run time such as $1.2 \mathrm{sn}$ for Example 3 and lower operation count results in reduction of cumulative truncation errors and improvement of overall accuracy. It follows from the numerical results that the accuracy of the solutions obtained using the operational method is quite good in comparison with the exact solution. Moreover, in Tables 3, we give the $L^{2}$-norm errors for Example 2.

Afterwards, the method can also be extended to the system of integro-differential equations, but some modifications are required.

Acknowledgement: Author is very grateful to the reviewers for their valuable comments and suggestions which has helped immensely in improving the quality of this manuscript.

\section{References}

[1] K. Parand, A. Pirkhedri, Sinc-collocation method for solving astrophysics equations, New Astronomy 2010, 15, 533-537

[2] M. Dehghan, F. Shakeri, Approximate solution of a differential equation arising in astrophysics using the variational iteration method, New Astronomy 2008, 13, 53-59.

[3] R. P. Agarwala, D. O'Reganb, Second order initial value problems of Lane-Emden type, Appl. Math. Lett. 2007, 20, 11981205.

[4] D.C.Biles, M.P. Robinson, J.S. Spraker, A generalization of the Lane-Emden equation, J. Math. Anal. Appl. 2002, 273, 654666.

[5] N.T. Shawagfeh, Nonperturbative approximate solution for Lane-Emden equation, J. Math. Phys. 1993, 34, 4364-4369.

[6] H.T. Davis, Introduction to nonlinear differential and integral equations, Dover, New York, 1962.

[7] H. Zou, A priori estimates for a semilinear elliptic system without variational structure and their applications, Mathematische Annalen 2002, 323, 713-735.

[8] J. Serin, H. Zou, Non-existence of positive solutions of the Lane-Emden system, Differential Integral Equa. 1996, 9, 635653.

[9] J. Serin, H. Zou, Existence of positive solutions of Lane-Emden systems, Atti del Sem. Mat. Fis. Univ. Modena 1998, 46, 369380.

[10] K.Parand, M. Dehghan, A. R. Rezaei, S. Ghaderi, An approximation algorithm for the solution of the nonlinear Lane-Emden type equations arising in astrophysics using Hermite functions collocation method, Comp. Phys. Comm. 2010, 181, 10961108. 
[11] O.P.Singh, R. K. Pandey, V. K. Singh, An analytic algorithm of Lane-Emden type equations arising in astrophysics using modified Homotopy analysis method, Comp. Phys. Comm. 2009, 180, 1116-1124.

[12] A.Yıldırım, T. Öziş, Solutions of singular IVPs of Lane-Emden type by the variational iteration method, Nonlinear Analysis 2009, 70, 2480-2484.

[13] Y.Q. Hasan, L. M. Zhu, Solving singular boundary value problems of higher-order ordinary differential equations by modified Adomian decomposition method, Commun. Nonlinear Sci. Numer. Simulat. 2009, 14, 2592-2596.

[14] R. K. Pandey, N. Kumar, A. Bhardwaj, G. Dutta, Solution of Lane-Emden type equations using Legendre operational matrix of differentiation, Appl. Math. Comp. 2012, 218(14), 76297637.

[15] E.H. Doha, W.M. Abd-Elhameed, Y.H. Youssri, Second kind Chebyshev operational matrix algorithm for solving differential equations of Lane-Emden type, New Astronomy 2013, 23-24, 113-117.

[16] Y.H. Youssri, W.M. Abd-Elhameed, E.H. Doha, Ultraspherical Wavelets method for solving Lane-Emden type equations, Rom. Journ. Phys. 2013, 60(9-10), 1298-1314.

[17] W.M. Abd-Elhameed, Y.H. Youssri, E.H. Doha, A novel operational matrix method based on shifted Legendre polynomials for solving second-order boundary value problems involving singular, singularly perturbed and Bratu-type equations, Math. Sci. 2015, 9, 93-102.

[18] W.M. Abd-Elhameed, E.H. Doha, Y.H. Youssri, New Spectral Second Kind Chebyshev Wavelets Algorithm for Solving Linear and Nonlinear Second-Order Differential Equations Involving Singular and Bratu Type Equations, Abstract and Applied Analysis 2013, Article ID:715756.

[19] B. Gürbüz, M. Sezer, Laguerre Polynomial Solutions of a Class of Initial and Boundary Value Problems Arising in Science and Engineering Fields, Acta Phys. Pol. A 2017, 132(3), 558-560.

[20] B. Bülbül Arslan, B. Gürbüz, M. Sezer, A Taylor matrixcollocation method based on residual error for solving LaneEmden type differential equations, New Trends in Mathematical Sciences 2015, 3(2), 219-224.

[21] M. Kumar, N. Singh, Modified Adomian Decomposition Method and computer implementation for solving singular boundary value problems arising in various physical problems, Comp. Chem. Eng. 2010, 34(11), 1750-1760.

[22] S.K.Varani, A. Aminataei, On the numerical solution of differential equations of Lane-Emden type. Comp. Math. Appl. 2010, 59, 2815-2820.

[23] D. Benko, D.C. Biles, M.P. Robinson, J.S. Spraker, Numerical approximation for singular second order differential equations, Math. Comp. Model. 2009, 49(5-6), 1109-1114.

[24] A. Aslanov, A generalization of the Lane-Emden equation, Int. Jour. Comp. Math. 2008, 85, 1709-1725.

[25] A. Yıldırım, T. Öziş, Solutions of singular IVPs of Lane-Emden type by homotopy perturbation method, Phys. Letters A 2007, 369, 70-76.
[26] A.M. Wazwaz, R. Rach, J.S. Duan, A study on the systems of the Volterra integral forms of the Lane-Emden equations by the Adomian decomposition method, Mathematical Methods in Applied Sciences 2013, 37(1).

[27] R. K. Pandey, N. Kumar, Solution of Lane-Emden type equations using Berstein operational matrix of differentiation, New Astronomy 2012, 17, 303-308.

[28] D. Flockerzi, K. Sundmacher, On coupled Lane-Emden equations arising in dusty fluidmodels. Journal of Physics: Conference Series 2011, 268, 012006.

[29] R. K. Pandey, N. Kumar, Solution of Lane-Emden type equations using Berstein operational matrix of differentiation, New Astronomy 2012, 17, 303-308.

[30] Y. Öztürk, M. Gülsu, An operational matrix method for solving Lane-Emden equations arising in astrophysics, Mathematical Methods in Applied Sciences 2014, 37, 2227-2235.

[31] J. C. Mason, D. C. Handscomb, Chebyshev polynomials, Chapman and Hall/CRC, New York, 2003.

[32] J. P. Body, Chebyshev and fourier spectral methods, University of Michigan, New York, 2000.

[33] W.M. Abd-Elhameed, E.H. Doha, Y.H. Youssri, M.A. Bassuony, New Tchebyshev Galerkin operational matrix method for solving linear and nonlinear hyperbolic telegraph type equations, Numerical Methods for Partial Differential Equations, 2016, 36(6), 1553-1571.

[34] A. Saadatmandi, Mehdi Dehghan, A new aperational matrix for solving fractional-order differential equations, Comp. Math. Appl. 2010, 59, 1326-1336.

[35] K. Parand, M. Razzaghi, Rational Chebyshev tau method for solving higher-order ordinary differential equations, Int. J. Comput. Math. 2004, 81, 73-80.

[36] E. Babolian, F. Fattahzadeh, Numerical solution of differential equations by using Chebyshev wavelet operational matrix of integration, Appl. Math. Comp. 2007, 188, 417-425.

[37] Y. Öztürk, M. Gülsu, Numerical solution of Abel equation using operational matrix method with Chebyshev polynomials, Asian-Europen Journal of Mathematics 2017, 10(3), 1750053.

[38] W.M. Abd-Elhameed, On solving linear and nonlinear sixthorder two point boundary value problems via an elegant harmonic numbers operational matrix of derivatives. CMES Comp. Model. Eng. Sci. 2014, 101(3), 159-185.

[39] K. Sayevand, K. Pichaghchi, A novel operational matrix method for solving singularly perturbed boundary value problems of fractional multi-order, International Journal of Computer Mathematics 2018, 95(4), 767-796.

[40] N. Irfan, S. Kumar, S. Kapoor, Bernstein Operational Matrix Approach for Integro-Differential Equation Arising in Control theory, Nonlinear Engineering: Modeling and Application 2014, 3(2), 117-123.

[41] M. Turkyilmazoglu, An effective approach for numerical solutions of high-order Fredholm integro-differential equations, Applied Mathematics and Computation 2014, 227, 384-398. 